\title{
Three-dimensional opto-fluidic devices fabricated by ultrashort laser pulses for high throughput single cell detection and processing
}

\author{
David J. Hwang • Moosung Kim • Kuniaki Hiromatsu • \\ Hojeong Jeon · Costas P. Grigoropoulos
}

Received: 7 January 2009 / Accepted: 3 March 2009 / Published online: 25 March 2009

(C) The Author(s) 2009. This article is published with open access at Springerlink.com

\begin{abstract}
Three-dimensional flow-through microchannels were fabricated inside bulk fused silica glass via ultrashort pulsed laser direct writing. The device fabrication sequence takes advantage of the nonlinear volumetric absorption in glass and the subsequent preferential chemical etching process. Optical waveguides were also written into the glass specimen and integrated with the fluidic conduits. Flow tests using both fluorescent particles and red blood cells (RBCs) were conducted on various three-dimensional channel configurations. Experiments showed the possibility for laser-induced cell processing inside the microchannels. To evaluate cytometer functionality, RBCs were detected inside the manufactured microchannel via both transmission and fluorescence probing.
\end{abstract}

PACS 42.62.-b $\cdot$ 42.62.Be $\cdot$ 81.05.kf $\cdot$ 87.17.uv $\cdot$ 42.55.wd

\section{Introduction}

Great attention has been paid to opto-fluidic circuit structures for cell-based life science research and diagnostics probing individual cells, since this is an essential step for cell manipulating, isolating and sorting. Opto-fluidic circuits have been suggested as promising tools for miniaturizing conventional cell detection devices. Such circuits are typically fabricated utilizing processes such as multistep lithography or imprinting. Variances employing dif-

D.J. Hwang · M. Kim · K. Hiromatsu $\cdot$ H. Jeon ·

C.P. Grigoropoulos $(\bowtie)$

Department of Mechanical Engineering, University of California,

Berkeley, CA 94720-1740, USA

e-mail: cgrigoro@me.berkeley.edu ferent materials have been proposed, including doped silicon dioxide waveguides connected to silicon microchannels [1], glass-based microfluidic devices fitted with inserted etched optical fibers [2], PDMS microfluidic channels integrated with PDMS waveguides of higher refractive index [3], and polymer-based microchannels coupled with SU-8 waveguides [4]. Reduction of the channel size in such devices is problematic due to clogging issues, hence increasing the complexity of single cell detection devices by requiring flow management concepts such as flow hydrodynamical [4, 5] or electrokinetical [2] focusing for uniformity of cell positioning in the channel. Additional schemes are also required for reaching single cell detection resolution, for example, via focusing incident light by micro-lens or signal processing via cross-correlation analysis.

The transfer of various exogenous substances such as genes, indicators, antibodies and macromolecules into vital cells is a crucial step in basic research, applied drug discovery and clinical gene therapy [6-8]. The most commonly adopted methods are chemical transfection [9], lipidbased [10], viral vector-based [11], electroporation [12] and laser-assisted photoporation [13, 14]. Efforts towards miniaturizing electroporation have adopted manipulation of microelectrodes, micropipette and capillaries [15] or microfluidic device-based methods [16, 17]. Laser-assisted photoporation has been demonstrated using various laser sources [18-20].

We have recently introduced [21] an opto-fluidic device incorporating three-dimensional flow-through microchannels inside bulk fused silica glass through ultrashort pulsed laser direct writing and subsequent chemical etching processes. The venturi-type microchannels have circular cross section and channel diameter of $\mathrm{O}(1-10 \mu \mathrm{m})$ at the flow neck and $\mathrm{O}(10-100 \mu \mathrm{m})$ upstream and downstream of the flow neck. The circular cross section of the flow channel 
neck is designed slightly smaller $(\sim 1-2 \mu \mathrm{m})$ than the targeted cell diameter, in order to snugly fit individual cells. This approach enables effectively "confocal" cell detection and processing at the microchannel neck. The incident light is guided by optical waveguides crossing the microchannel and can probe local optical property disturbance, enabling single cell detection by analyzing the respective transmission intensity change. The guided incident light also can be used to locally excite fluorescence from a dyed cell that passes through the microchannel neck, enabling another scheme of single cell detection by detecting fluorescence emission light collected by optical waveguide. Glass is a desirable material for biological applications because it offers excellent optical, chemical, biological and thermal stability. Because of the device transparency, structural integrity and monolithic structure, cell treatment concepts can be easily incorporated into the device, including laser-based single cell processing. Site-specific injection can be realized by incorporating additional three-dimensional crossing microchannels on the neck region. Optical processing of cells has been demonstrated taking advantage of the superb optical transparency through the fabricated microchannels.

\section{Fabrication of three-dimensional microchannels}

Figure 1 shows the fabrication sequence of the threedimensional microchannels. Femtosecond laser microprocessing is an attractive tool to fabricate three-dimensional microchannels taking unique advantage of highly confined volumetric absorption via nonlinear MPA (Multi-Photon Absorption) processes in virtually all kinds of transparent materials. A number of efforts on fabricating threedimensional microchannels inside silica glass have been carried out. Even though direct machining of three-dimensional microchannels has been demonstrated [22], special care was

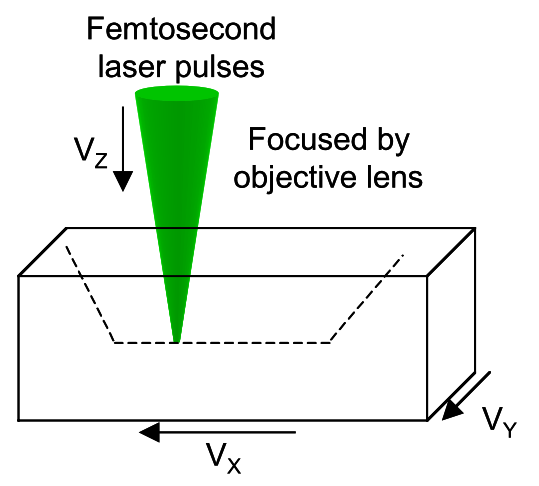

Fig. 1 Three-dimensional microchannel fabrication via direct writing of the desired microchannel pattern by femtosecond laser pulses. Subsequent chemical wet etching process in a HF solution assisted by ultrasonic agitation was used to define the microchannels needed for efficient debris removal and the optical transparency was not optimal following the ablation process. Consequently, the laser parameters were maintained in the glass melting regime. The subsequent re-solidification induced a structural change through glass transition. In this regime, the modified region experiences an optical refractive index change that is typically used to fabricate threedimensional optical waveguides [23, 24]. Since the modified region inside glass has orders of magnitude higher etch rate when exposed to HF solution, subsequent wet etching can define through-channels [25]. High repetition rate fiber laser $(1 \mathrm{MHz}, \sim 500$ fs pulse duration, pulse energy of 150-200 nJ and $522 \mathrm{~nm}$ wavelength) was used for stable three-dimensional processing, assisted by thermal accumulation effects inside the glass [26]. The laser beam was focused through an objective lens $(\mathrm{NA}=0.55)$ to generate arbitrary three-dimensional channels via precise 3 -axis motorized stage translation. Figure 2(a) shows a side view optical image of a single microchannel formed inside the glass specimen. Further examples of arbitrary microchannel manifolds are displayed in Fig. 2(b) $-(\mathrm{g})$. The diameter of the microchannel neck can be adjusted to $\sim 5-30 \mu \mathrm{m}$ depending on etching time and laser scanning parameters, while a neck length even smaller than the neck diameter can be achieved, representing an ideal configuration for the proposed work.

In Fig. 3(a)-(c), transmission optical images of $\sim 500 \mathrm{~nm}$ particle suspension (Fig. 3(a)), diluted red blood cell (RBC) solution (Fig. 3(b)), and high concentration RBC solution (Fig. 3(c)) are captured by regular CCD camera, showing superb optical transparency and excellent flow characteristics through the hydrophilic glass channels. An example of fluorescence imaging is also shown in Fig. 3(d), by using a suspension of fluorescing particles ( $\sim 1 \mu \mathrm{m}$ diameter). Pneumatic relative pressure control was performed to regulate the flow velocity and assist the precise cell positioning.

\section{Optical cell processing through the fabricated microchannels}

Fused silica is transparent to laser radiation over a wide spectral range from ultraviolet (UV) to near-infrared (IR) and of either continuous wave or short temporal pulse width. Taking advantage of the excellent optical transparency through the fabricated three-dimensional microchannels, various laser conditions can be applied on the proposed channel structures aiming at detailed and specific cell processing. In Fig. 4, direct focusing of amplified femtosecond laser pulses $(\sim 100$ fs pulse duration, $800 \mathrm{~nm}$ wavelength and $1 \mathrm{~mJ}$ maximum pulse energy, $1 \mathrm{kHz}$ maximum) through the proposed microchannel was performed. Initially, the laser focus was adjusted based on laser-induced bubble generation in the pure liquid. The laser pulse energy was then reduced below the liquid phase change threshold. 


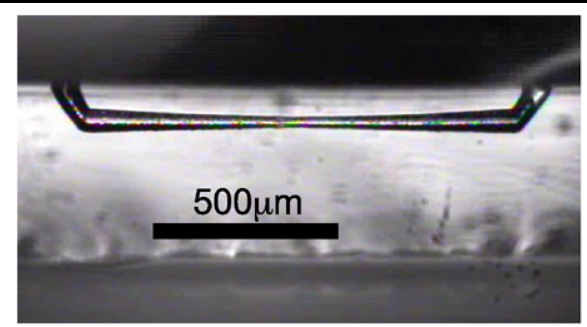

a

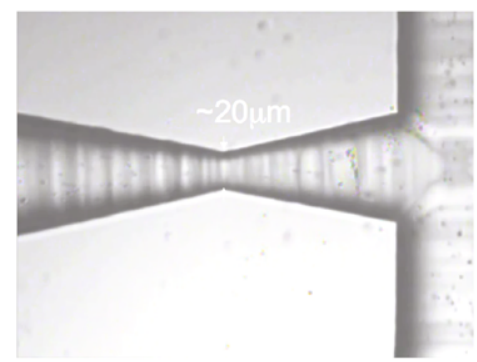

e

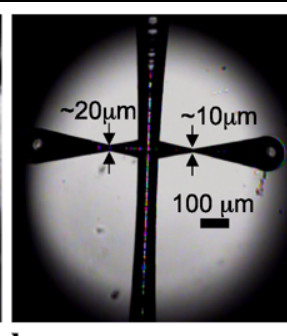

b

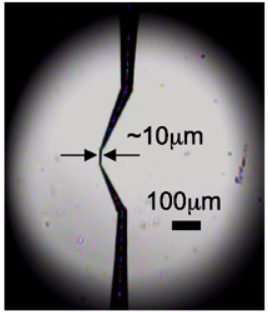

c

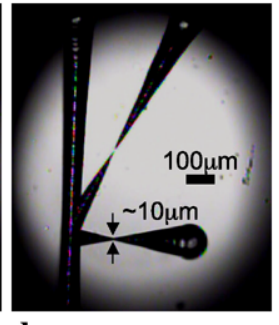

d

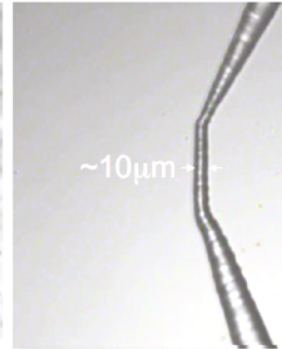

$\mathbf{f}$

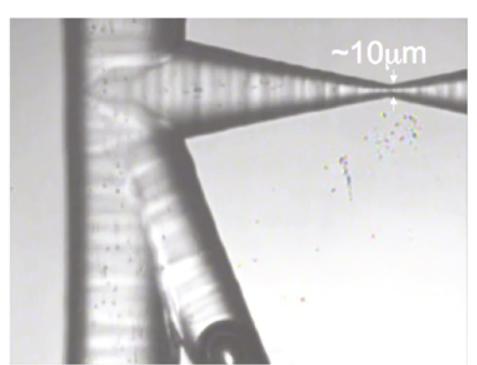

g

Fig. 2 Examples of fabricated three-dimensional microchannels. a Micro-through-channel, b-d variations of microchannel design to shape the neck region. $\mathbf{e}-\mathbf{g}$ depict magnified views

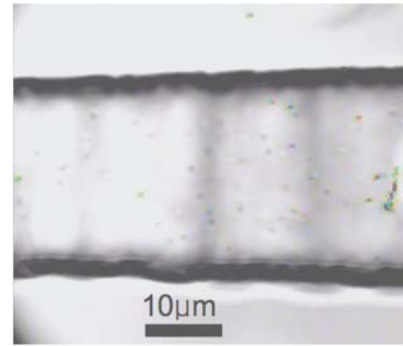

a

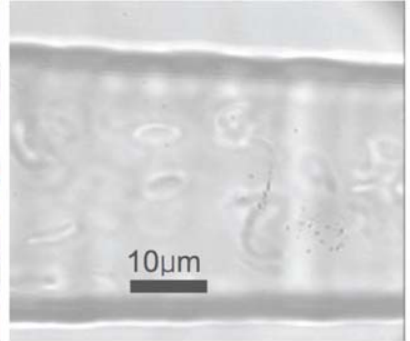

b

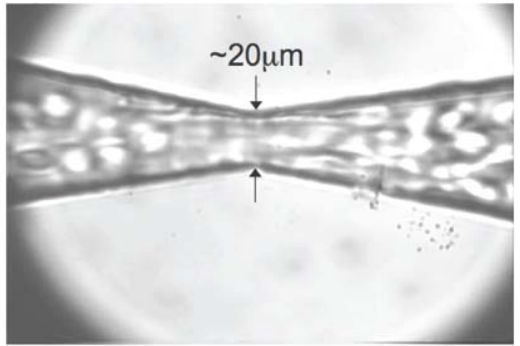

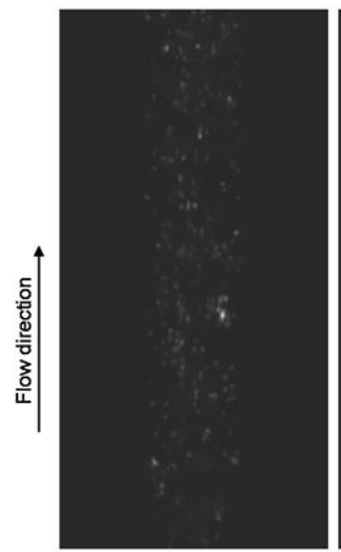
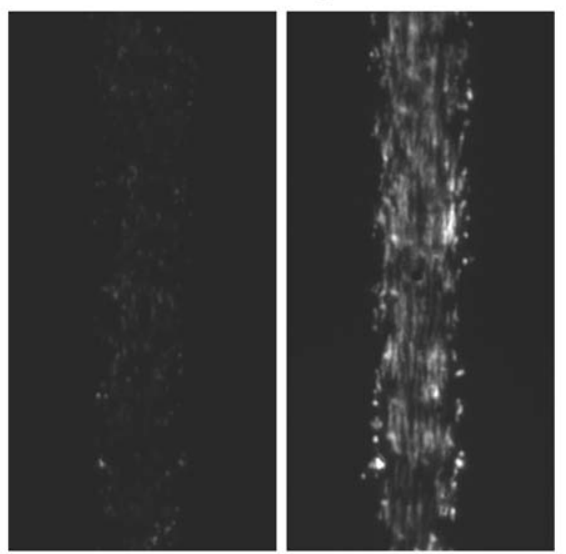
d

Fig. 3 Flow imaging examples through three-dimensional microchannels. a $\sim 500 \mathrm{~nm}$ particle solution, b diluted red blood cell (RBC) solution, $\mathbf{c}$ high concentration RBC solution, and $\mathbf{d}$ imaging of flow by

When dilute red blood cell suspension was flown under preadjusted laser conditions (not incurring liquid ablation in the matrix liquid), a laser-induced bubble was generated only $\sim 1 \mu \mathrm{m}$ fluorescing particles. Exposure time was $1 \mathrm{~ms}$. Different images in $\mathbf{d}$ correspond to different flow velocities as controlled by pneumatic pressure control

when a RBC cell was within the laser focus, indicating interaction with a minute part of the RBC cell (laser focal spot is around $2 \mu \mathrm{m}$ ). After termination of the laser pulse, 


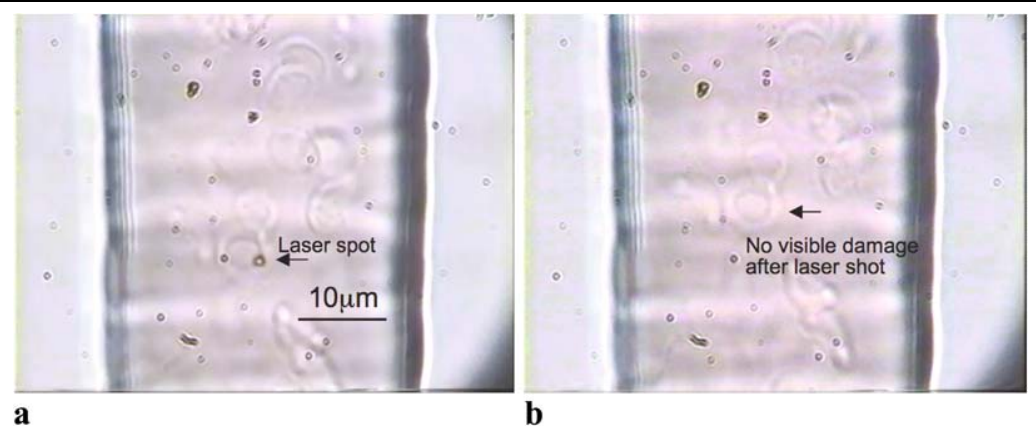

Fig. 4 Laser-based cell processing example inside microchannels fabricated in glass. a A single femtosecond laser pulse of $\sim 100 \mathrm{fs}, 800 \mathrm{~nm}$ wavelength and $\sim 100 \mathrm{~nJ}$ pulse energy was focused by an objective lens of NA $=0.55$. Under this laser condition, laser-induced bubble appeared only when the red blood cell was within the laser focus and b after termination of laser pulse, no visible damage occurred on the RBC

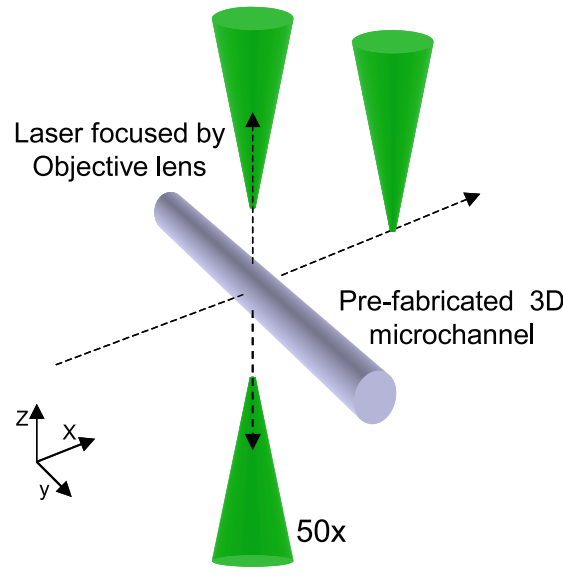

a

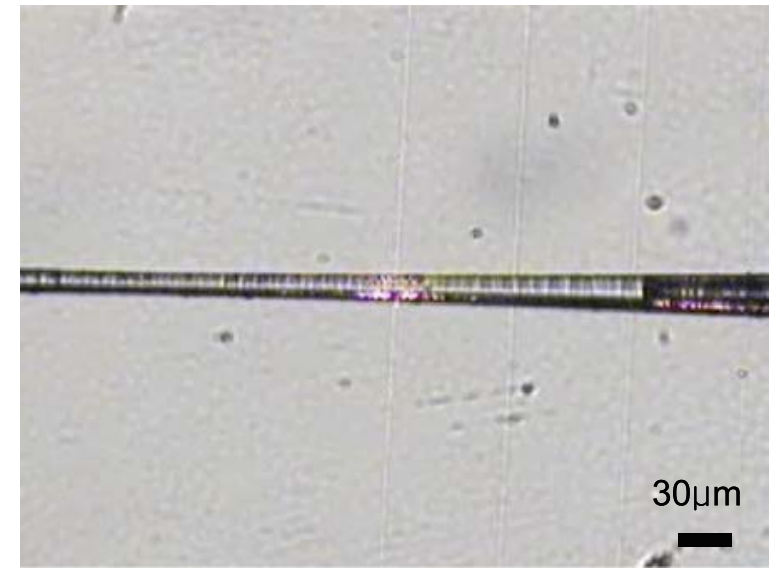

b
Fig. 5 Fabrication of three-dimensional optical waveguides. a Schematic of laser direct writing to fabricate optical waveguide, crossing pre-fabricated microchannel and $\mathbf{b}$ examples of combined mi-

the generated bubble vanished and no visible damage was inspected on the cell. This situation should be close to the desirable site-specific cell photoporation, showing compatibility of the proposed microchannel with precise laser-based cell processing. However, further work is needed to demonstrate the photoporation efficacy by direct observation.

\section{Fabrication of combined three-dimensional optical waveguide structures}

Combined three-dimensional microchannel and waveguide structures have been successfully demonstrated [21]. In Fig. 5(a), the fabrication process of three-dimensional optical waveguides is outlined. After completing threedimensional microchannel fabrication, laser direct writing as shown in Fig. 1(a) can generate combined optical waveguides crossing the pre-fabricated microchannels. As crochannel and optical waveguides. The HeNe laser was coupled to one of the waveguides, showing local illumination inside the microchannel

shown in Fig. 5(b), a HeNe laser (632.8 nm) beam was successfully coupled through the waveguide structure, illuminating locally the microchannel.

For further performance evaluation of the combined structures, cell counting experiments were carried out using dilute red blood cells (Fig. 6). A HeNe laser beam coupled through an illumination waveguide intercepted the fluidic microchannel and was then transmitted through a collection waveguide and collected by an optical detector. Fluorescence signal-based counting of $\sim 1 \mu \mathrm{m}$ fluorescence particles was also reported [21] and compared to tests by a commercial cytometer. The measured counting density followed the trend of the calculated counting density, verifying that the proposed glass-based opto-fluidic device can effectively function as a cell/particle counter or pre-detector of single cell manipulation on a bio-chip. The operation range also can be extended up to the order of cell counting efficiency of commercial cytometer via fine tuning of the detection electric circuit and particle speed calibration. Considering the 


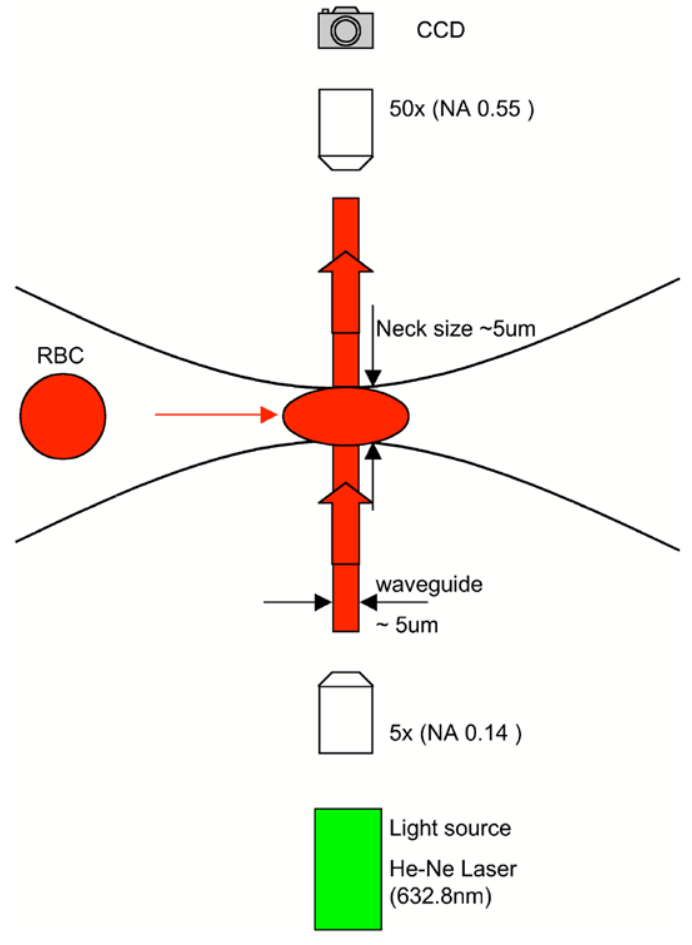

Fig. 6 Cell counting experiment by proposed combined threedimensional microchannel and optical waveguide structures. Diluted red blood cell suspension is driven by controlled flow through the mi-

significant efforts to realize combined opto-fluidic devices for biological Lab-on-a-chip applications, the present results prove the massive scale capability of localized illumination and in-situ monitoring of the cell conditions. Additionally, fluorescence schemes as implemented in [21] provide detection specificity.

It is further argued that the combined optical waveguide structures can assist more stable photoporation processes through "confocal" illumination of the laser beam onto the cell membrane as well as automated cell processing in combined flow-through channels. Through careful adjustment of the crossing waveguide size and layout, alternative sitespecific photoporation schemes can be examined.

\section{Conclusion}

Glass-based opto-fluidic devices integrating microchannels and optical waveguides were fabricated by femtosecond laser pulses and subsequent wet etching. Use of microchannels with a neck diameter slightly smaller than the cell size enables sharp, constant and unambiguous cell detection via transmission and reflection as well as cell processing via coupling laser radiation.

Fusion of the glass-based opto-fluidic device with microfabricated components will expand its potential as a micrototal analysis system ( $\mu$ TAS). New concepts of single cell

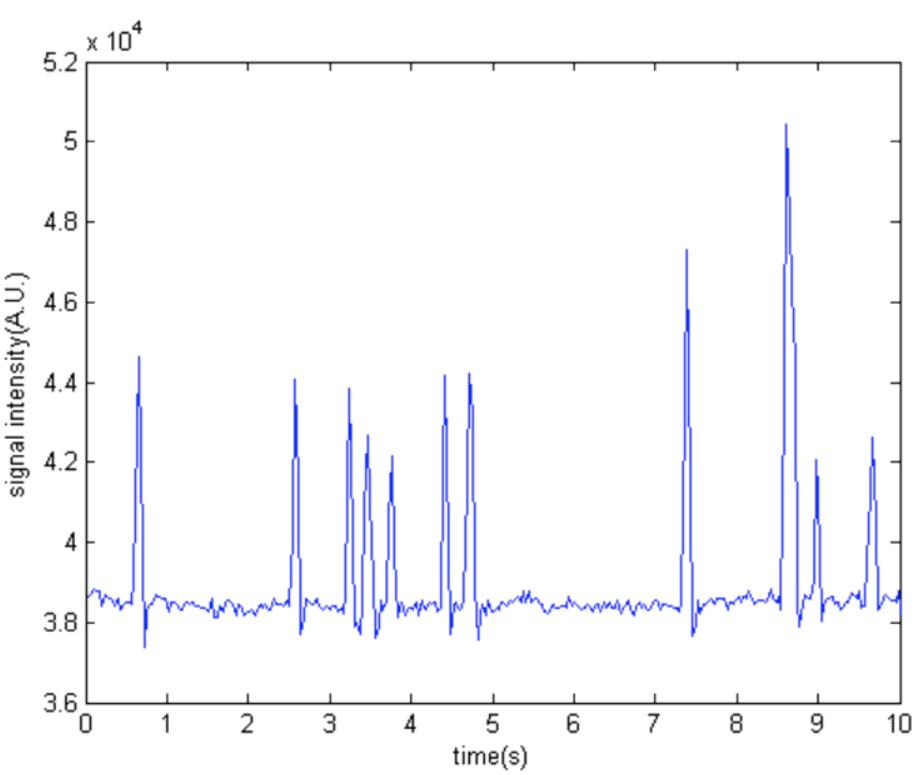

crochannel. A HeNe laser was coupled to the illumination waveguide and collected through the collection waveguide

treatment in microchannels can be applied due to the tapered circular shape of the glass channel that is configured capture a single cell at a time. Therefore, the femtosecond laser 3-D direct writing in fused silica and the successful integration of fluidic microchannels and optical waveguides offer a new pathway to future generation of biochip devices.

Acknowledgement Support by the U.S. National Science Foundation under grant DMI-0556363 is gratefully acknowledged.

Open Access This article is distributed under the terms of the Creative Commons Attribution Noncommercial License which permits any noncommercial use, distribution, and reproduction in any medium, provided the original author(s) and source are credited.

\section{References}

1. P. Friis, K. Hoppe, O. Leistiko, K.B. Mogensen, J. Hubner, J.P. Kutter, Appl. Opt. 40, 6246 (2001)

2. L.M. Fu, R.J. Yang, C.H. Lin, Y.J. Pan, G.B. Lee, Anal. Chim. Acta 507, 163 (2004)

3. V. Lien, K. Zhao, Y.H. Lo, Appl. Phys. Lett. 87, 194106 (2005)

4. Z. Wang, J. El-Ali, M. Engelund, T. Gotsaed, I.R. Perch-Nielsen, K.B. Mogensen, D. Snakenborg, J.P. Kutter, A. Wolff, Lab Chip 4, 372 (2004)

5. C.H. Lin, G.B. Lee, J. Micromech. Microeng. 13, 447 (2003)

6. S. Mehier-Humbert, R.H. Guy, Adv. Drug Deliv. Rev. 57, 733 (2005)

7. F. Stracke, I. Rieman, K. König, J. Photoch. Photobiol. B 81, 136 (2005) 
8. I.B. Clark, E.G. Hanania, J. Stevens, M. Gallina, A. Fieck, R. Brandes, B.O. Palsson, M.R. Koller, J. Biomed. Opt. 11, 014034 (2006)

9. F.L. Graham, A.J. Van der Eb, A new technique for the assay of infectivity of human adenovirus 5 DNA. Virology 52, 456 (1973)

10. R. Fraley, S. Subramani, P. Berg, D. Papahadjopoulos, J. Biol. Chem. 255, 431 (1980)

11. D.A. Rubinson, C.P. Dillon, A.V. Kwiatkowski, C. Sievers, L. Yang, J. Kopinja, D.L. Rooney, M. Zhang, M.M. Ihrig, M.T. McManus, F.B. Gertler, M.L. Scott, L. Van Parijs, Nat. Genet. 33, 401 (2003)

12. E. Neumann, M. Schaefer-Ridder, Y. Wang, P.H. Hofschneider, EMBO J. 1, 841 (1982)

13. W. Tao, J. Wilkinson, E.J. Stanbridge, M.W. Berns, Proc. Natl. Acad. Sci. 84, 4180 (1987)

14. U.K. Tirlapur, K. König, Nature 418, 290 (2002)

15. K. Nolkrantz, C. Farre, A. Brederlau, R.I.D. Karlsson, C. Brennan, P.S. Eriksson, S.G. Weber, M. Sandberg, O. Orwar, Anal. Chem. 73, 4469 (2001)

16. Y.C. Lin, C.M. Jen, M.Y. Huang, C.Y. Wu, X.Z. Lin, Sens. Actuators B 79, 137 (2001)

17. Y.S. Shin, K. Cho, J.K. Kim, S.H. Lim, C.H. Park, K.B. Lee, Y. Park, C. Chung, D. Han, J.K. Chang, Anal. Chem. 76, 7045 (2004)
18. L. Paterson, B. Agate, M. Comrie, R. Ferguson, T.K. Lake, J.E. Morris, A.E. Carruthers, C.T.A. Brown, W. Sibbett, P.E. Bryant, F. Gunn-Moore, A.C. Riches, K. Dholakia, Opt. Express 13, 595 (2005)

19. D. Stevenson, B. Agate, X. Tsampoula, P. Fischer, C.T.A. Brown, W. Sibbett, A. Riches, F. Gunn-Moore, K. Dholakia, Opt. Express 14, 7125 (2006)

20. I. Turovets, A. Lewis, D. Palanker, H. Gilo, A. Vilenz, J.C. Broder, S. Lewis, Biotechniques 15, 1022 (1993)

21. M. Kim, D.J. Hwang, H. Jeon, K. Hiromatsu, C.P. Grigoropoulos, Lab Chip 9, 311 (2009)

22. D. Hwang, T. Choi, C.P. Grigoropoulos, Appl. Phys. A 79, 605 (2004)

23. K.M. Davis, K. Miura, N. Sugimoto, K. Hirao, Opt. Lett. 21, 1729 (1996)

24. M. Will, S. Nolte, B.N. Chichkov, A. Tünnermann, Appl. Opt. 41, 4360 (2002)

25. C. Hnatovsky, R.S. Taylor, P.P. Rajeev, E. Simova, V.R. Bhardwaj, D.M. Rayner, P.B. Corkum, Appl. Phys. Lett. 87, 014104 (2005)

26. L. Shah, A. Arai, S. Eaton, P. Herman, Opt. Express 13, 1999 (2005) 